\title{
Analysis of CSMA/CA mechanism of IEEE 802.15.6 under non-saturation regime
}

\author{
Saeed Rashwand, Jelena Mišić, Senior Member, IEEE, and Vojislav B. Mišić, Senior Member, IEEE
}

\begin{abstract}
We have developed an analytical model for a non-saturated IEEE 802.15.6 wireless body area network (WBAN) operating under an error-prone channel. The most suitable vehicle for improving network performance was found to be the choice of access phase lengths based on traffic loads for different User Priorities (UPs). It was also found that the deployment of exclusive access phase (EAP) is not necessary in a typical WBAN; in fact, short exclusive and random access phases (EAP and RAP, respectively) lead to inefficient use of available bandwidth. We have also found that four user priorities (out of the eight available) typically suffice to achieve even the most stringent requirements for WBAN performance.
\end{abstract}

Index Terms-wireless body area networks (WBANs); IEEE 802.15.6; probabilistic analysis; access priorities

\section{INTRODUCTION}

Recent technological advances have led to the development of low cost, low power sensing devices that allow continuous tracking of patient's vital health variables through wireless body area networks (WBANs) [1], [2]. In this manner, healthcare providers can react appropriately when necessary, and also collect a rich history of patient health data.

WBANs have been built using proprietary MAC and PHY protocols [3], [4], as well as existing WLAN/WPAN standards such as IEEE 802.11, IEEE 802.15.4, ZigBee, and Bluetooth [5], [6]. IEEE has recently published the IEEE 802.15.6 standard [7] that combines low power operation and simplified CSMA-CA similar to IEEE 802.15.4, whilst providing a prioritization mechanism, similar to IEEE 802.11e, which is well suited for transmission of healthcare data. However, in-depth performance analyses of IEEE 802.15.6 CSMA/CA mechanism are still scarce. Initial research work in this direction focused on the network operating in saturation regime which can be analyzed with a discrete-time Markov chain (DTMC) only, without queuing analysis of the node buffer (which is always full) and the associated extension to a semi-Markov chain with general distribution of time between state transitions. In [8], [9], a numerical model was developed to evaluate the theoretical throughput and delay limits of IEEE 802.15.6-based networks, however the model does not consider user priorities and access phases of the standard, and

- S. Rashwand, J. Mišić and V. B. Mišić are with the Department of Computer Science, Ryerson University, Toronto, Ontario. assumes a collision-free network over an ideal channel. In [10], [11], authors studied the performance of the IEEE 802.15.6 MAC under saturation condition for all traffic classes, while the impact of access phases lengths was studied in [12].

However, saturation leads to excessive delays, reduction in throughput, and instability, if the buffers are assumed to be of infinite size. Thus, stable operation of a WBAN necessitates that its nodes operate well below saturation. In this case, CSMA/CA performance has been investigated in [13], [14] for IEEE 802.11, [15], [16], [17] for IEEE 802.11e, and [18] for IEEE 802.15.4, typically using a combination of queuing and Markov chain analysis. However, these models are not directly applicable to the analysis of IEEE 802.15.6 networks due to the differences in their respective implementations of the CSMA/CA mechanism. A simulation-only study of 802.15.6 networks has been reported in [19], while [21] investigates the performance of IEEE 802.15.6-based networks under nonsaturation condition, albeit without considering the impact of queuing on the departure process, which leads to oversimplification. Our earlier work in [20] focused on the impact of fading in physical layer and the interaction between physical and MAC layer using a simplified MAC model of IEEE 802.15.6 CSMA/CA mechanism. Models in [10], [11] do not consider queuing analysis and probabilistic backoff duration sub-models integrated in a semi-Markov process framework.

In this work, we present a detailed analytical model for investigating the performance of the IEEE 802.15.6-based WBANs. The model includes a Geo/G/1 queuing sub-model of the node buffer, and addresses all eight UPs $\left(\mathrm{UP}_{k}, k=0 \ldots 7\right)$ and the first exclusive and random access phases (EAP1 and RAP1) under finite load and an error prone channel. This model is solved iteratively to obtain the main performance indicators of the network, such as mean waiting time and transmission success probability. We have also developed a complete simulation model, first of its kind, using OPNET Wireless Modeler [23] to validate the analytical model.

The paper is organized as follows: Section 2 briefly introduces the IEEE 802.15.6 standard. In Section 3, we describe the major features of the analytical model. In Section 4, we analyze the performance of an IEEE 802.15.6-based WBAN for all UPs. Section 5 summarizes our findings and concludes the paper. An Online Supplement presents the findings regarding the network that uses RTS/CTS handshake. 


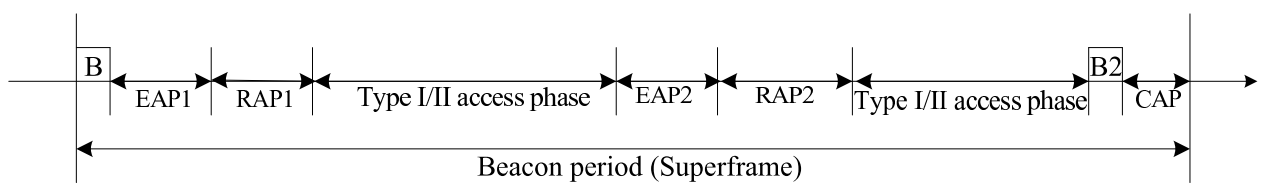

Fig. 1. Layout of access phases in a superframe.

\section{IEEE 802.15.6 STANDARD}

The IEEE 802.15.6 standard defines no less than eight user priorities (UPs) which are differentiated based on the minimum and maximum contention windows, $C W_{\min }$ and $C W_{\max }$, respectively, as shown in Table 1.

TABLE 1

BAN User Priority Mapping

\begin{tabular}{cccc} 
UP & Traffic designation & $C W_{\min }$ & $C W_{\max }$ \\
\hline 0 & Background (BK) & 16 & 64 \\
1 & Best effort (BE) & 16 & 32 \\
2 & Excellent effort (EE) & 8 & 32 \\
3 & Controlled load (CL) & 8 & 16 \\
4 & Video (VI) & 4 & 16 \\
5 & Voice (VO) & 4 & 8 \\
6 & Media data or network control & 2 & 8 \\
7 & Emergency or medical event report & 1 & 4
\end{tabular}

The time axis is divided into beacon periods (superframes) by the coordinator of the network. A superframe may include exclusive access phases (EAP1 and EAP2), random access phases (RAP1 and RAP2), type-I/II access phases, and contention access phase (CAP), in the order shown in Fig. 1; all phases except RAP1 may have a zero length. The EAP periods can be only accessed for transmitting the $\mathrm{UP}_{7}$ frames while RAP and CAP periods can be used by all UPs. To improve channel utilization, a node with $\mathrm{UP}_{7}$ may treat the combined EAP1 and RAP1 as a single EAP1, and the combined EAP2 and RAP2 as a single EAP2. Type-I/II access phases are utilized by the hub for the polling mechanism; other phases are contention-based access phases. In this work, we focus on EAP1 and RAP1 phases, and set the lengths of all the access phases to zero.

A detailed description of the pertinent aspects of operation of an IEEE 802.15.6 network can be found in the standard [7].

\section{Analytical Model}

In this section, we outline the analytical model for investigating the performance of the CSMA mechanism of IEEE 802.15.6. The analytical model consists of three inter-related sub-models: Markov chain sub-model, sub-model of backoff durations, and queuing sub-model. The notation for the most important parameters is shown in Table 2.

In all three models, we consider that a node of $\mathrm{UP}_{k}$, has a single queue of user priority $k$. (In all subsequent discussions, we will assume that $k=0 \ldots 7$, unless otherwise indicated.) The network, operating in $2.4 \mathrm{GHz}$ ISM band, is assumed to be single hop including a hub as the coordinator and $n_{k}$ nodes of $\mathrm{UP}_{k}$ and therefore we ignore the hidden terminal problem. We assume that there is only uplink traffic from the nodes to the hub. Let $\lambda_{k}$ denote the data frame arrival probability during a CSMA slot for a node of $\mathrm{UP}_{k}$. We assume that during a slot at most one data frame arrives to the queue which is not an unrealistic assumption due to the small length of a CSMA slot. It is clear that the inter-arrival time $I_{k}$ is geometrically distributed with the mean of $\frac{1}{\lambda_{k}}$ slots. The lengths of EAP1 and RAP1 in slots are denoted with eap and rap, respectively. We assume that the size of the beacon is small, hence we ignore it in our analytical model.

The control frames and headers are transmitted at $91.4 \mathrm{kbps}$ while we assume the payload of the data frames is transmitted at $971.6 \mathrm{kbps}$. The size of the data frames for a node of $\mathrm{UP}_{k}$ is denoted by $l_{k}$ (in slots) and $l_{k, b}$ (in bits), while ack and $a c k_{b}$ denote the size of an ACK frame in slots and in bits, respectively.

We assume an error-prone channel having the Bit Error Rate (BER) of ber, in which case $\sigma_{k}=(1-b e r)^{l_{k, b}+a c k_{b}}$ denotes the probability that the data frame and the corresponding acknowledgement are transmitted without getting corrupted by the noise.

\subsection{Markov Chain Sub-model}

We observe the system at moments of beginning of EAPs and RAPs, backoff decrements, and packet departures. In these points, referred to as Markov points, the random process under study has a Markov property. The time interval between two successive Markov points is a random variable which affects the steady state probabilities. Therefore, the process under consideration is a semi-Markov process [24]. For evaluating the semi Markov process we need DTMCs and distribution of time between the Markov points. The Markov chain submodel is composed of a set of eight 3-dimensional DTMCs to compute the access probabilities of the UPs. The DTMCs are developed based on the backoff procedure of the IEEE 802.15.6 CSMA mechanism. This sub-model also requires the probability that the queue is empty after a data frame service completion, which is calculated in the queuing sub-model.

The Markov Chain sub-model actually consists of eight 3-dimensional DTMCs, one for each UP, to formulate the medium access probabilities of their respective UPs. A UP $k$ Markov chain represents the transitions among the backoff states of a $\mathrm{UP}_{k}$ node. All those Markov chains are interdependent since each of them must take into account not only medium access probabilities, idle queue probabilities, and backoff durations of the corresponding node, but also those of other nodes in the network.

The access probability of a $\mathrm{UP}_{k}$ node, $\tau_{k}$, is calculated by solving the set of DTMCs considering the CSMA slots in which the medium is not set to be busy due to either a 
TABLE 2

Important parameter notation ( $k$ denotes user priority for a node).

\begin{tabular}{|c|c|c|c|}
\hline Parameter & Description & Parameter & Description \\
\hline$k$ & Index of UP & $\sigma_{k}$ & $\begin{array}{l}\text { Probability that neither a data frame nor its ACK is } \\
\text { corrupted by noise }\end{array}$ \\
\hline$n_{k}$ & Number of nodes of $\mathrm{UP}_{k}$ & $\lambda_{k}$ & Data frame arrival probability during a CSMA slot \\
\hline$\tau_{k}$ & Medium access probability & $g_{k}$ & Probability that medium is idle during backoff countdown \\
\hline$L_{k, s}$ & Successful data frame transmission time & $L_{k, c}$ & Unsuccessful data frame transmission time \\
\hline$L_{k, s o}$ & $\begin{array}{l}\text { Mean successful data frame transmission time of other } \\
\text { nodes }\end{array}$ & $L_{k, c o}$ & $\begin{array}{l}\text { Mean unsuccessful data frame transmission time of other } \\
\text { nodes }\end{array}$ \\
\hline$\eta_{k}$ & Probability of successful medium access & $p_{k}$ & $\begin{array}{l}\text { Probability that there is not enough time to complete a } \\
\text { data frame transmission from the current CSMA slot to } \\
\text { the end of the RAP1 period }\end{array}$ \\
\hline$\pi_{k, 0}^{\prime}$ & Probability of an empty queue after serving a data frame & $p_{k, I d l e}$ & Probability of being in the idle state in a CSMA slot \\
\hline$p_{s o, k}$ & $\begin{array}{l}\text { Probability of a successful transmission by the other } \\
\text { nodes }\end{array}$ & $p_{c o, k}$ & $\begin{array}{l}\text { Probability of an unsuccessful transmission by the other } \\
\text { nodes }\end{array}$ \\
\hline$\Phi_{k}(z)$ & PGF for the duration of backoff process & $\Pi_{k}(z)$ & $\begin{array}{l}\text { PGF of steady state probability distribution of number of } \\
\text { frames in the queue after completing data frame service }\end{array}$ \\
\hline$\omega_{k}$ & Mean waiting time for a data frame & $\zeta_{k}$ & Mean response time for a data frame \\
\hline
\end{tabular}

transmission on the medium or being in an inaccessible access phase. The access probabilities vary in different time periods. We calculate the access probabilities in the CSMA slots in which the medium is accessible, using the DTMC for a node of $\mathrm{UP}_{k}$ shown in Fig. 2. The Markov points are beginning of the CSMA slots in which the $\mathrm{UP}_{k}$ node is allowed to transmit. Hence, the intervals between two successive Markov points in a DTMC may have different lengths. The Markov chain represents a random process with stationary distribution $b_{k, i, j}$, where $k$ denotes the user priority of the node, $i=0 \ldots R$ denotes the backoff phase in the backoff procedure invoked by the node, and $j=0 \ldots W_{k, i}$ denotes the value of the backoff counter.

After developing all the DTMCs, the Markov chains are solved as a single system to calculate the medium access probabilities.

Let $f=\prod_{i=0}^{7}\left(1-\tau_{i}\right)^{n_{i}}$ denote the probability that a CSMA slot is idle during a RAP period. Then, the probability that the medium remains idle during the backoff countdown of a $\mathrm{UP}_{k}$ node, $k \leq 6$, is $f_{k}=\frac{f}{1-\tau_{k}}$. However, $\mathrm{UP}_{7}$ nodes are the only ones allowed to transmit during EAP1 while all the UPs can access the medium during RAP1, and the probabilities that the medium remains idle in a CSMA slot during EAP1 and RAP1 are different. The corresponding probability for a node of $\mathrm{UP}_{7}$ is

$$
f_{7}=\frac{X_{R} f}{\left(X_{E}+X_{R}\right)\left(1-\tau_{7}\right)}+\frac{X_{E}\left(1-\tau_{7}\right)^{n_{7}-1}}{X_{E}+X_{R}}
$$

where $X_{E}$ and $X_{R}$ denote the mean number of CSMA slots in EAP1 and RAP1, respectively. Their initial values are set to $X_{E}=e a p$ and $X_{R}=r a p$, and then updated in subsequent iterations via

$$
\begin{aligned}
X_{R}^{\prime} & =\frac{\operatorname{rap}-L_{7, s}}{f+\sum_{t=0}^{7} n_{t} \tau_{t} \eta_{t} L_{t, s}+\left(1-f-\sum_{t=0}^{7} n_{t} \tau_{t} \eta_{t}\right) L_{t, c}} \\
X_{E}^{\prime} & =\frac{\operatorname{eap}}{\chi+n_{7} \tau_{7} \psi \sigma_{7} L_{7, s}+\left(1-\chi-n_{7} \tau_{7} \psi \sigma_{7}\right) L_{7, c}}
\end{aligned}
$$

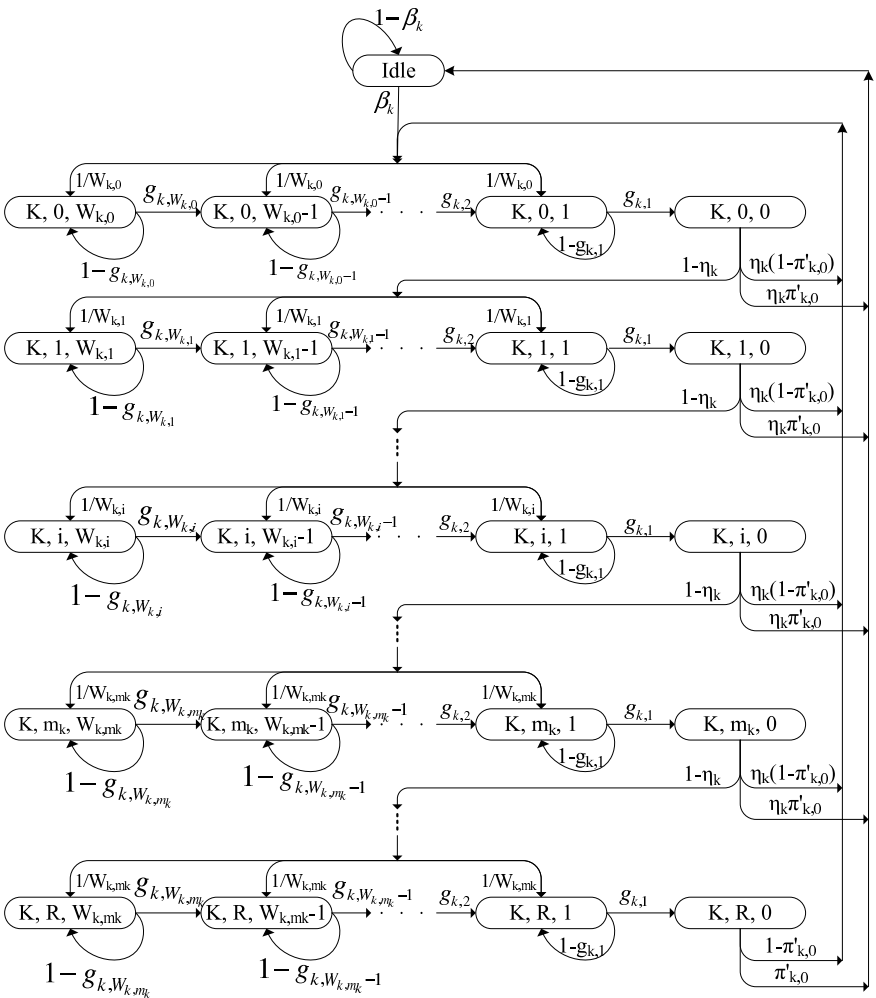

Fig. 2. Developed 3-dimensional DTMC for $\mathrm{UP}_{k}$.

In the equations above, $L_{k, x}=l_{k}+a c k+$ sifs denotes the time needed for a data frame transmission, with subscripts $s$ and $c$ used to distinguish between successful and unsuccessful transmissions, respectively. Furthermore, $L_{k, x o}=l_{k, o}+a c k+$ sifs denotes the corresponding transmission time of other nodes (denoted with the subscript 'o') in the network, where $l_{k, o}$ is the average size (in slots) of a data frame transmitted by those other nodes for a given $\mathrm{UP}_{k}$ node.

Initial values for $l_{k, o}$ are set to

$$
l_{k, o}=\frac{\sum_{u=0}^{7} n_{u} l_{u}-l_{k}}{\sum_{u=0}^{7} n_{u}-1}
$$

where $\chi=\left(1-\tau_{7}\right)^{n_{7}}$ and $\psi=\left(1-\tau_{7}\right)^{n_{7}-1}$. 
and then updated in each subsequent iteration as

$$
l_{k, o}^{\prime}=\frac{\sum_{u=0}^{7} n_{u} \tau_{u} l_{u}-\tau_{k} l_{k}}{\sum_{u=0}^{7} n_{u} \tau_{u}-\tau_{k}}
$$

In the Markov chain shown in Fig. 2, $\eta_{k}=f_{k} \sigma_{k}$ represents the probability of successful access to the medium for a $\mathrm{UP}_{k}$ node when its backoff counter reaches zero.

However, if the remaining time during the current access phase (EAP1 and RAP1 for a $\mathrm{UP}_{7}$ node, and RAP1 otherwise) is not long enough to complete a data frame transmission, the backoff counter is locked; for a node without $\mathrm{UP}_{7}$ the counter must be kept locked during EAP1 as well.

The backoff counter remains locked until the moment when the node is allowed to transmit (RAP1 for $\mathrm{UP}_{k}, k \leq 6$ or EAP1 for $\mathrm{UP}_{7}$ ). Then, the probability that in a given CSMA slot the node notices that there is not enough time during the current access period to complete transmission, is

$$
p_{k}= \begin{cases}\frac{1}{r a p-L_{k, s}-C_{k}}, & k \leq 6 \\ \frac{1}{\text { rap }+ \text { eap }-L_{k, s}-C_{7}}, & k=7\end{cases}
$$

where $C_{k}=\frac{C W_{k, \min }+C W_{k, \max }}{4}$ approximates the mean backoff value. The number of accessible CSMA slots in a superframe for a given UP (the denominator in the equation above) depends on the length of accessible access phases, frame transmission time, and the backoff counter at which the counter is paused.

The probability that the backoff counter of a node with $\mathrm{UP}_{k}$ is decremented (i.e., not locked) upon reaching $j=1 . . W_{k, m_{k}}$ is

$$
g_{k, j}=f_{k}\left(1-p_{k} \frac{1-f_{k}^{j}}{1-f_{k}}\right)
$$

The slots in which the backoff counter is locked are still considered as Markov points in order to calculate the access probability, which for a $\mathrm{UP}_{k}$ node, is $\tau_{k}=\sum_{i=0}^{R} b_{k, i, 0}$.

If the input probability to the zero-th backoff phase is

$$
Y_{k}=\frac{\tau_{k} \eta_{k}\left(1-\pi_{k, 0}^{\prime}\right)+p_{k, \text { Idle }} \beta_{k}}{1-\left(1-\eta_{k}\right)^{R+1}\left(1-\pi_{k, 0}^{\prime}\right)}
$$

the solution of the Markov chain will give us

$$
\begin{aligned}
b_{k, i, j} & =\frac{\left(1-\eta_{k}\right)^{i} Y_{k}\left(W_{k, i}-j+1\right)}{W_{k, i} g_{k, j}} \\
b_{k, i, 0} & =\left(1-\eta_{k}\right)^{i} Y_{k}
\end{aligned}
$$

The probability of being in "Idle" state is

$$
p_{k, \text { Idle }}=\frac{\tau_{k} \eta_{k} \pi_{k, 0}^{\prime}}{\beta_{k}\left(1-\left(1-\eta_{k}\right)^{R+1}\right)}
$$

The probability that a data frame arrives to the queue during the time interval between two successive Markov points is

$$
\begin{aligned}
\beta_{k} & =f_{k} p_{k}\left(1-\left(1-\lambda_{k}\right)^{L_{k}}\right)+f_{k}\left(1-p_{k}\right) \lambda_{k} \\
& +\left(1-f_{k}\right)\left(p_{k} \sum_{u=0}^{L_{k, s o}-1}\left(1-p_{k}\right)^{u}\left(1-\left(1-\lambda_{k}\right)^{L_{k}+u+1}\right)\right. \\
& \left.+\left(1-p_{k}\right)^{L_{k, s o}}\left(1-\left(1-\lambda_{k}\right)^{L_{k, s o}}\right)\right)+\frac{1}{x_{k}}
\end{aligned}
$$

where $x_{k}=X_{R}, k \leq 6$, and $x_{7}=X_{R}+X_{E} \cdot \pi_{k, 0}^{\prime}$ is the probability that the queue of the node with $\mathrm{UP}_{k}$ is empty when a data frame is either successfully transmitted or dropped due to an exceeded retry limit, which will be calculated in the queuing sub-model.

In the last equation, the first component corresponds to the case where at the first CSMA slot of the interval no node transmits on the medium but there is not enough time to complete a data frame transmission; this causes the backoff counter to lock until the next superframe. In this case, the length of the interval is $L_{k}$ slots. During RAP1 all the nodes in the network have to lock their backoff counters; while during EAP1, $U P_{7}$ nodes are allowed to transmit or decrease their backoff counters. Consequently, $L_{k}=$ eap + sifs $+l_{k}+a c k, k \leq 6$, but $L_{7}=$ sifs $+l_{k}+a c k$.

The second component represents the case where the first CSMA slot remains idle and there is enough time to complete a data frame transmission. This results in the interval length of one slot.

The third component corresponds to the case where a node starts transmitting; $\frac{1}{x_{k}}$ is the probability that medium access is not possible in the first CSMA slot.

We compute the sum of all stationary distribution $b_{k, i, j}$ as

$$
\sum_{i=0}^{R} \sum_{j=0}^{W_{k, i}} b_{k, i, j}=Y_{k} \sum_{i=0}^{R}\left(1-\eta_{k}\right)^{i}\left(1+\sum_{j=1}^{W_{k, i}} \frac{W_{k, i}-j+1}{W_{k, i} g_{k, j}}\right)
$$

The sum of probabilities must be one, which results in the following set of equations for $k=0 \ldots 7$ :

$$
1=p_{k, \text { Idle }}+Y_{k} \sum_{i=0}^{R}\left(1-\eta_{k}\right)^{i}\left(1+\sum_{j=1}^{W_{k, i}} \frac{W_{k, i}-j+1}{W_{k, i} g_{k, j}}\right)
$$

Hence, the Markov chain sub-model results in a set of eight equations with unknown $\tau_{k}$ and $\pi_{k, 0}^{\prime}, k=0 \ldots 7$, the latter set being obtained from the queuing sub-model.

\subsection{Sub-model of Backoff Duration}

The probability distribution of all the backoff phases and the total backoff duration before a successful access to the medium or a data frame drop for all UPs are formulated by the backoff duration sub-model. The probability generating functions (PGFs) of the backoff durations are computed based on the access probabilities of the UPs which are introduced in the Markov chain sub-model. The computed time distributions are then substituted in the queueing and the Markov sub-models to calculate the transition probabilities in the corresponding semi-Markov chains.

To calculate the length of backoff durations we extend the DTMCs described above so that the beginning of every CSMA slot represents a Markov point; as the result, any two consecutive Markov points are separated by a single slot. To compose the extended 4-dimensional DTMCs we replace the component shown on top of Fig. 3 with the component shown in the 3-dimensional DTMCs (Fig. 2) for $k=0 . .7, i=0 \ldots R$, and $j=1 \ldots W_{k, i}$.

Stationary distributions of $b_{k, i, j, S, t}, t=1 \ldots L_{k, s o}$, and $b_{k, i, j, C, t}, t=1 \ldots L_{k, c o}$, correspond to the time periods 


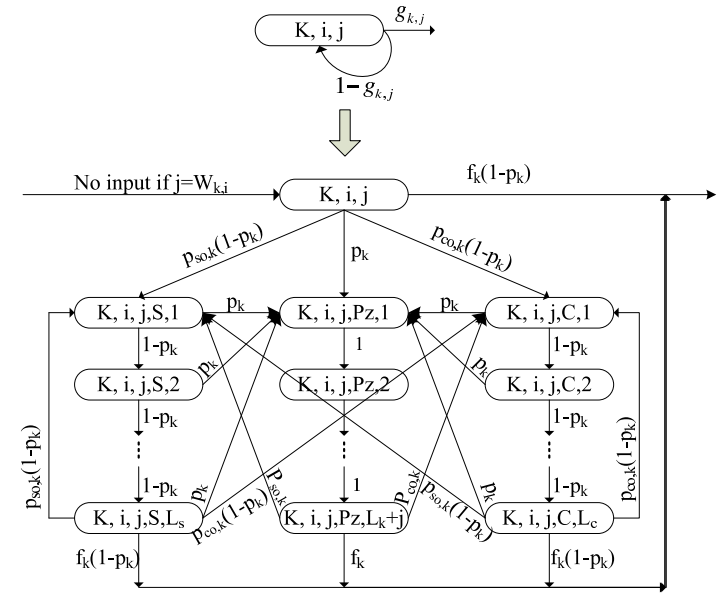

Fig. 3. Markov chain state extension for $\mathrm{UP}_{k}$

in which the data frame is (not) successfully transmitted, while stationary distribution of $b_{k, i, j, P z, t}$ for $t=1 \ldots L_{k}+j$ corresponds to the time period in which the node backoff counter is locked on account of insufficient time to complete a transmission. As before, subscripts $s$ and $c$ and their uppercase counterparts denote successful and unsuccessful medium accesses, respectively, while $P z$ is used to label the pause caused by inability to access the medium.

We refer to $p_{s o, k}$ and $p_{c o, k}$ as the probabilities that the medium becomes busy due to a successful transmission by another node and unsuccessful accesses by the other nodes in the network, respectively, which are computed as:

$$
\begin{aligned}
& p_{s o, k}=\sum_{i=0}^{7} \frac{n_{i} \tau_{i} \eta_{k}}{1-\tau_{i}}-\frac{\tau_{k} \eta_{k}}{1-\tau_{k}} \\
& p_{c o, k}=1-f_{k}-p_{s o, k}
\end{aligned}
$$

where $\frac{\tau_{i} \eta_{k}}{1-\tau_{i}}$ represents the probability of successful transmission by a $\mathrm{UP}_{i}$ node. We note that $p_{s o, k}+p_{c o, k}=1-f_{k}$ is the probability that the medium is busy due to an ongoing transmission.

Let us denote the PGFs for the duration of a data frame (successful or unsuccessful) transmission as $\operatorname{St}_{k}(z)=C t_{k}(z)=$ $z^{l_{k}+a c k+s i f s}$. Every backoff period is composed of up to $R$ backoff phases. The backoff phases are made of the time periods in which the backoff counter is decremented by one until the counter reaches zero.

Let $\mathcal{B}_{S, k, j}(z)\left(\mathcal{B}_{C, k, j}(z)\right)$ denote the PGFs for the time interval during which the backoff counter is locked due to successful (unsuccessful) medium access by other nodes, which may be calculated from the extended DTMCs, as follows:

$$
\begin{aligned}
\mathcal{B}_{P, k, j}(z) & =z^{L_{k}+j}\left(f_{k} z+\Theta_{k, j}(z)\right) \\
\mathcal{B}_{S, k, j}(z) & =p_{k} \mathcal{B}_{P, k, j}(z) \frac{1-\left(1-p_{k}\right)^{L_{k, s o}} z^{L_{k, s o}}}{1-\left(1-p_{k}\right) z} \\
& +\left(1-p_{k}\right)^{L_{k, s o}} f_{k} z^{L_{k, s o}}+\left(1-p_{k}\right)^{L_{k, s o}} \Theta_{k, j}(z)
\end{aligned}
$$

Also, let $\mathcal{B}_{P, k, j}(z)$ denote the PGF for the time interval during which the backoff counter is locked due to insufficient time to complete a transmission, which may be calculated as

$$
\begin{aligned}
\mathcal{B}_{C, k, j}(z) & =p_{k} \mathcal{B}_{P, k, j}(z) \frac{1-\left(1-p_{k}\right)^{L_{k, c o}} z^{L_{k, c o}}}{1-\left(1-p_{k}\right) z} \\
& +\left(1-p_{k}\right)^{L_{k, c o}} f_{k} z^{L_{k, c o}}+\left(1-p_{k}\right)^{L_{k, c o}} \Theta_{k, j}(z)
\end{aligned}
$$

where $\Theta_{k, j}(z)=p_{s o, k} \mathcal{B}_{S, k, j}(z)+p_{c o, k} \mathcal{B}_{C, k, j}(z)$ is PGF of the mean duration of backoff counter lock due to a transmission on the medium, either successful or unsuccessful.

The values of $\mathcal{B}_{S, k, j}(z), \mathcal{B}_{C, k, j}(z)$, and $\mathcal{B}_{P, k, j}(z)$ can be obtained using the substitution method of solving system of equations. We now calculate the PGF of the time interval between the moment when the backoff counter of a node of $\mathrm{UP}_{k}$ becomes $j$ and the moment when the backoff counter becomes $j-1$ at the $i$-th backoff phase. The PGF of the time to decrease the backoff counter by one, where the counter is $j$, is given by

$$
\Phi_{k, i, j}(z)=p_{k} \mathcal{B}_{P, k, j}(z)+\left(1-p_{k}\right)\left(f_{k} z+\Theta_{k, j}(z)\right)
$$

for $k=0 \ldots 7, j=1 \ldots W_{k, i}$, and $i=0 \ldots R$.

Using the above PGFs we can write the PGF of $i$-th backoff phase duration as

$$
\begin{aligned}
\Phi_{k, i}(z) & =\sum_{j=1}^{W_{k, i}} \prod_{t=1}^{j} \Phi_{k, i, t}(z)\left(\eta_{k}\left(L_{k, s} p_{k} z^{L_{k}}+1-L_{k, s} p_{k}\right)\right. \\
& \left.+\left(1-\eta_{k}\right)\left(L_{k, c} p_{k} z^{L_{k}}+1-L_{k, c} p_{k}\right)\right) / W_{k, i}
\end{aligned}
$$

The PGF for the duration of backoff process for a $\mathrm{UP}_{k}$ node is

$$
\begin{aligned}
\Phi_{k}(z) & =\sum_{i=0}^{m_{k}}\left(\prod_{u=0}^{i} \Phi_{k, u}(z)\right)\left(1-\eta_{k}\right)^{i}\left(z^{L_{k, c}}\right)^{i} \eta_{k} \\
& +\sum_{i=m_{k}+1}^{R}\left(\prod_{u=0}^{m_{k}} \Phi_{k, u}(z)\right) \Phi_{k, m_{k}}^{i-m_{k}}(z)\left(1-\eta_{k}\right)^{i}\left(z^{L_{k, c}}\right)^{i} \eta_{k} \\
& +\left(\prod_{u=0}^{m_{k}} \Phi_{k, u}(z)\right) \Phi_{k, m_{k}}^{R-m_{k}}(z)\left(1-\eta_{k}\right)^{R+1}\left(z^{L_{k, c}}\right)^{R+1}
\end{aligned}
$$

From (19), we calculate the mean backoff time before the medium is successfully accessed or the data frame is dropped for a $\mathrm{UP}_{k}$ node as $\phi_{k}=\left.\frac{d}{d z} \Phi_{k}(z)\right|_{z=1}$.

\subsection{Queuing Sub-model}

To calculate durations of idle periods and access probabilities of all UPs we need to know the queuing status of each UP in each CSMA slot. The backoff probability distributions of all UPs, acquired by the backoff duration sub-model, are then used to arrive at the empty queue probability, duration of an idle period, and the queue size of every UP.

We model the queue of each node in the WBAN using a Geo/G/1 system with vacations [26]. The inter-arrival time to the queue for all UPs is geometrically distributed with mean of $\frac{1}{\lambda_{k}}$ slots. A data frame is serviced when it is successfully transmitted or dropped due to an exceeded retry limit. The time interval in which the node performs the backoff process or is idle because of an empty queue is called a vacation. We assume that upon a successful access to the medium each node transmits a single data frame. 
Let $B_{k}(z)=\Phi_{k}(z) \operatorname{St}_{k}(z)$ denote the PGF of service time (in slots) of a data frame for a $\mathrm{UP}_{k}$ node, which includes the backoff process and successful transmission time. The mean service time for a $\mathrm{UP}_{k}$ data frame is $b_{k}=B_{k}^{\prime}(1)$.

The offered load or traffic intensity of the queue is given by $\rho_{k}=\lambda_{k} b_{k}$. For stability condition the offered load must be less than one, in which case the offered load is equal to the carried load or the server utilization in the Geo/G/1 system.

We consider a Markov chain $\left\{D_{k, n} ; n=0,1, \ldots\right\}$, where $D_{k, n}$ is the number of data frames present in the system for a $\mathrm{UP}_{k}$ node immediately after the service completion of the $n$ th data frame. We refer to $A_{k, n}$ as the number of data frames that arrive during the service of the $n$-th data frame of a $\mathrm{UP}_{k}$ node. If the number of data frames of $\mathrm{UP}_{k}$ that arrive during an idle period is also denoted by $\alpha_{k}$, we have

$$
D_{k, n+1}= \begin{cases}\alpha_{k}+A_{k, n+1}-1, & \text { if } D_{k, n}=0 \\ D_{k, n}+A_{k, n+1}-1, & \text { otherwise }\end{cases}
$$

The PGF for the number of data frames that arrive during service time is $A_{k}(z)=B_{k}\left[\Lambda_{k}(z)\right]$, where $\Lambda_{k}(z)=1-\lambda_{k}+\lambda_{k} z$ is the PGF of the number of $\mathrm{UP}_{k}$ data frames that arrive during a single CSMA slot. Likewise, the PGF of the number of data frames that arrive during idle time is $\alpha_{k}(z)=\mathcal{I}_{k}\left(\Lambda_{k}(z)\right)$, where $\mathcal{I}_{k}(z)$ is the PGF of the idle period duration - the difference between the inter-arrival time and the frame service time. Since the $\mathrm{UP}_{k}$ data frame arrival rate is $\lambda_{k}$, the number of idle slots before an arrival follows a geometric distribution with the PGF of $\frac{\lambda_{k} z}{1-\left(1-\lambda_{k}\right) z}$. However, the service time of the last served data frame must be deducted from the inter-arrival time, hence

$$
\mathcal{I}_{k}(z)=\frac{\lambda_{k} z}{B_{k}(z)\left(1-\left(1-\lambda_{k}\right) z\right)}
$$

Let the steady state probability of having $u \geq 0$ frames in the $\mathrm{UP}_{k}$ queue after service completion be

$$
\pi_{k, u}=\pi_{k, 0} \sum_{j=1}^{u+1} \alpha_{k, j} a_{k, u-j+1}+\sum_{j=1}^{u+1} \pi_{k, j} a_{k, u-j+1}
$$

where $A_{k}(z)=\sum_{u=0}^{\infty} a_{k, u} z^{u}$ and $\alpha_{k}(z)=\sum_{u=0}^{\infty} \alpha_{k, u} z^{u}$. The PGF of the steady state probability distribution $\left\{\pi_{k, u} ; u=\right.$ $0,1, \ldots\}$ for the queue of a $\mathrm{UP}_{k}$ node is given by

$$
\begin{aligned}
\Pi_{k}(z)= & \sum_{u=0}^{\infty} \pi_{k, u} z^{u} \\
= & \sum_{u=0}^{\infty} \pi_{k, 0} \sum_{j=1}^{u+1} \alpha_{k, j} a_{k, u-j+1} z^{u} \\
& +\sum_{u=0}^{\infty} z^{u} \sum_{j=1}^{u+1} \pi_{k, j} a_{k, u-j+1} \\
= & \pi_{k, 0} \sum_{j=1}^{\infty} \alpha_{k, j} z^{j} \sum_{u=j-1}^{\infty} a_{k, u-j+1} z^{u-j+1} \\
& +\sum_{j=1}^{\infty} \pi_{k, j} z^{j-1} \sum_{k=j-1}^{\infty} a_{k, u-j+1} z^{u-j+1}
\end{aligned}
$$

which ultimately gives

$$
\Pi_{k}(z)=\frac{\pi_{k, 0}\left(1-\mathcal{I}_{k}\left(\Lambda_{k}(z)\right)\right) B_{k}\left(\Lambda_{k}(z)\right)}{B_{k}\left(\Lambda_{k}(z)\right)-z}
$$

The probability that the buffer is empty (after a successful data frame transmission or a data frame drop due to an exceeded retry limit) is determined from the normalization condition as

$$
\pi_{k, 0}=\frac{1-\rho_{k}}{E\left[\alpha_{k}\right]}=\frac{1-\rho_{k}}{\lambda_{k} E\left[\mathcal{I}_{k}(z)\right]}
$$

The probability $\pi_{k, 0}$ depends on the time interval between two successive successful transmissions/data frame drops, which in turn depends on the background traffic, the contention among the nodes, and the data frame arrival rates. The backoff duration of a node is a function of the backoff durations and frame arrival rates of other nodes in the network which might be at different backoff phases at different time slots. Hence, $\pi_{k, 0}$ represents an average probability.

From (13) and (24) we obtain a set of 16 equations which enables us to calculate the 16 unknown variables of $\tau_{k}$ and $\pi_{k, 0}$, for $k=0 \ldots 7$. To solve this model analytically, we employ an iterative approach. We first solve the model based on initial values for $X_{E}, X_{R}$, and $f_{7}$. The next iterations are performed using the computed values in equations (2) and (1) based on the data calculated in the previous iteration. Iteration $t+1, t \leq 0$ is computed based on the outputs of iteration $t$ and the computed values in equations (2) and (1). The iterations are repeated until the value difference between two successive $X_{R}$ values is less than a predefined value (0.1), up to a maximum of 6 iterations.

The PGF of waiting time for a $\mathrm{UP}_{k}$ data frame, defined as the time interval from the moment when the frame arrives to the moment when its successful transmission begins, is

$$
\Omega_{k}(z)=\left\{\begin{array}{lr}
\Theta_{k}(z)(\theta x+1-\theta), & k \leq 6 \\
\Theta_{7}(z), & \text { otherwise }
\end{array}\right.
$$

where the term

$$
\Theta_{k}(z)=\frac{\left(1-\rho_{k}\right)\left(1-\mathcal{I}_{k}(z)\right)\left(1-\Lambda_{k}\left[B_{k}(z)\right]\right)}{E\left[\mathcal{I}_{k}(z)\right] \lambda_{k}\left[1-B_{k}(z)\right]\left(\Lambda_{k}\left[B_{k}(z)\right]-z\right)} \Phi_{k}(z)
$$

represents the PGF of the waiting time of the $\mathrm{UP}_{k}$ data frame until it reaches the top of the queue [26], while $\theta=\frac{e a p^{2}}{2(e a p+r a p)}$ corresponds to the PGF of the waiting time from the moment when the data frame reaches to the top of the queue until the moment when its transmission backoff process begins; $\Phi_{k}(z)$ is the PGF of the backoff duration of the data frame.

By solving the set of sub-models for all UPs, we are able to compute the mean waiting time of a $\mathrm{UP}_{k}$ data frame as

$$
\omega_{k}=\left\{\begin{array}{l}
\frac{\lambda_{k} b_{k}^{(2)}-\lambda_{k} b_{k}}{2\left(1-\rho_{k}\right)}+\frac{E\left[\mathcal{I}_{k}(z)\left(\mathcal{I}_{k}(z)-1\right)\right]}{2 E\left[\mathcal{I}_{k}(z)\right]}+\theta+\phi_{k}, k \leq 6 \\
\frac{\lambda_{k} b_{k}^{(2)}-\lambda_{k} b_{k}}{2\left(1-\rho_{k}\right)}+\frac{E\left[\mathcal{I}_{k}(z)\left(\mathcal{I}_{k}(z)-1\right)\right]}{2 E\left[\mathcal{I}_{k}(z)\right]}+\phi_{k}, k=7
\end{array}\right.
$$

where $b_{k}^{(2)}=b_{k}+\left.\frac{d^{2}}{d z^{2}} B_{k}(z)\right|_{z=1}$. Finally, the mean response time, defined as the time interval from frame arrival to the time it leaves the system, is $\zeta_{k}=\omega_{k}+\left(l_{k}+\right.$ sifs $\left.+a c k\right)$. 
TABLE 3

Experimental setup: traffic parameters.

\begin{tabular}{|c|c|c|c|c|c|c|}
\hline UP & data stream & number of nodes & traffic load $(\mathrm{p} / \mathrm{s})$ & packet size (Bytes) & sampling rate $(\mathrm{Hz})$ & sample size (bits) \\
\hline \multirow[t]{2}{*}{7} & $\mathrm{ECG}$ & 1 & 2 & 150 & 200 & 12 \\
\hline & EEG & 1 & 2 & 150 & 200 & 12 \\
\hline 6 & ECG & 2 & 2 & 150 & 200 & 12 \\
\hline \multirow[t]{2}{*}{5} & EEG & 1 & 2 & 150 & 200 & 12 \\
\hline & Blood Pressure & 1 & 2 & 150 & 200 & 12 \\
\hline \multirow[t]{4}{*}{4} & Glucose & 1 & 1 & 50 & 50 & 16 \\
\hline & Oxygen Saturation & 1 & 1 & 50 & 50 & 16 \\
\hline & Temperature & 1 & 0.25 & 20 & 5 & 8 \\
\hline & Respiration Rate & 1 & 0.25 & 20 & 5 & 8 \\
\hline 3 & Physical Activity & 4 & 1 & 50 & 50 & 16 \\
\hline 2 & EMG & 1 & 4 & 500 & 1000 & 16 \\
\hline 1 & $\mathrm{ECG}$ & 5 & 0.8 & 375 & 200 & 12 \\
\hline 0 & EEG & 8 & 0.5 & 600 & 200 & 12 \\
\hline
\end{tabular}

\section{Performance Evaluation}

We have used Maple 13 [27] to solve the analytical model outlined above and to calculate the performance descriptors of the network. Moreover, we have developed a simulation model of the IEEE 802.15.6 in OPNET [23]; this model implements the complete functionality of IEEE 802.15 .6 as stipulated by the standard.

The wireless healthcare network which is used for performance evaluation of the IEEE 802.15.6 standard under non-saturation regime consists of a hub and 28 nodes. The nodes include ten channels for Electroencephalogram (EEG), eight channels for Electrocardiogram (ECG), one blood pressure sensor, one glucose monitoring node, one blood oxygen saturation monitoring sensor (pulse oximeter), four physical activity monitoring sensors, one channel for Electromyogram $(E M G)$, one body temperature sensor, and one respiration rate monitoring node.

The nodes are categorized into eight UPs, as shown in Table 3 which uses the parameter values from [28]. We assume that all the nodes in a given UP have equal traffic load and payload size.

While this setup may be somewhat arbitrary from the perspective of a healthcare worker, it is fairly representative of a real scenario in which an IEEE 802.15.6 WBAN might be employed.

We set the differentiation parameters of $C W_{k, \min }$ and $C W_{k, \max }$ for all the nodes according to the standard, as shown in Table 1 . The retry limit is set to $R=7$ for all the UPs.

\subsection{Medium access}

Fig. 4 show mean waiting time for select UPs in the scenario RAP1 length varies while the EAP1 length is constant. As before, some UPs are omitted for clarity, and analytical and simulation results are shown with lines and crosses, respectively.

Results for the scenario in which the length of EAP1 varies from 0.05 second to 0.12 second while the length of RAP1 is fixed at 0.3 second are shown in Fig. 5 (as before, simulation results are omitted for clarity). The results confirm that increasing the length of EAP1, under non-saturation condition, increases the mean waiting time of the data frames, specifically

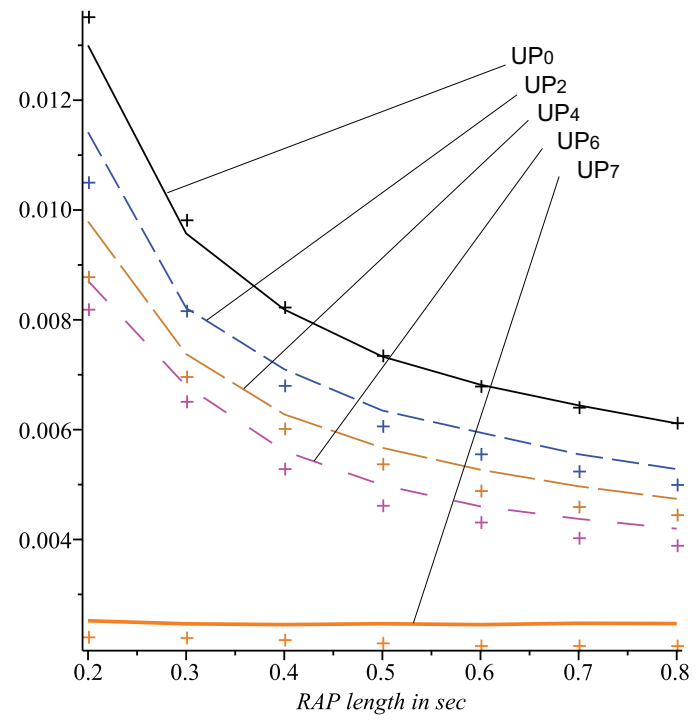

Fig. 4. Mean waiting time: all UPs, length of EAP1 is 0.05 sec.

the data frames that do not belong to the highest priority traffic class $\mathrm{UP}_{7}$.

\subsection{Effectiveness of user priorities}

We have also investigated the effectiveness of user priorities provided by the IEEE 802.15.6 standard. We consider a network with medium load, consisting of a number of nodes with identical traffic loads of two packets per second with the packet payload of 150B, and vary the number of nodes whilst keeping other parameters unchanged.

In the first scenario, we consider a WBAN with four nodes in each of the eight UPs. Fig. 6(a) show the mean waiting time for select UPS. As can be seen, the difference between all categories except $\mathrm{UP}_{7}$ (the highest one) is rather small, despite quite different contention window sizes. As before, lines denote analytical results, while crosses denote simulation results.

This conclusion is confirmed by the second experiment in which the WBAN uses only four UPs consisting of eight nodes each. Comparing the results in Fig. 6(a) and Fig. 6(b), we may 


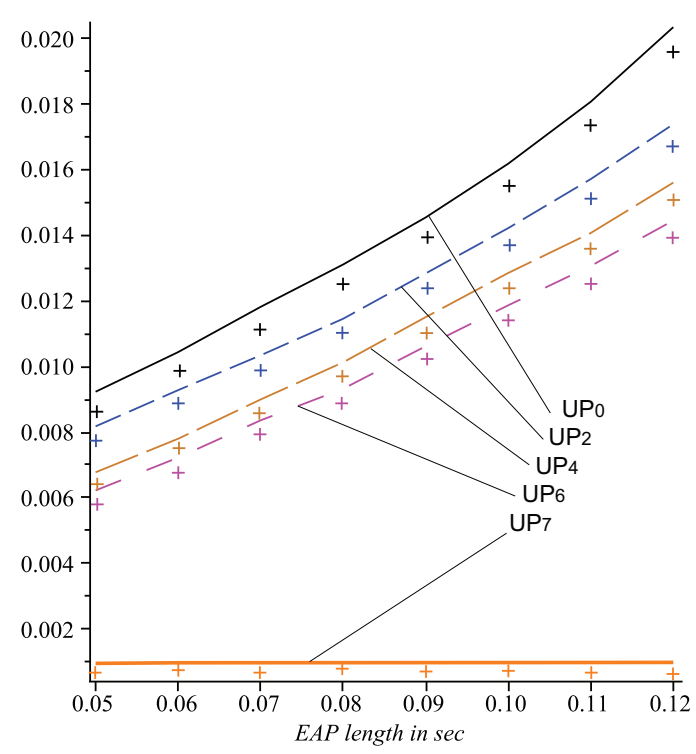

(a) Mean Waiting Time

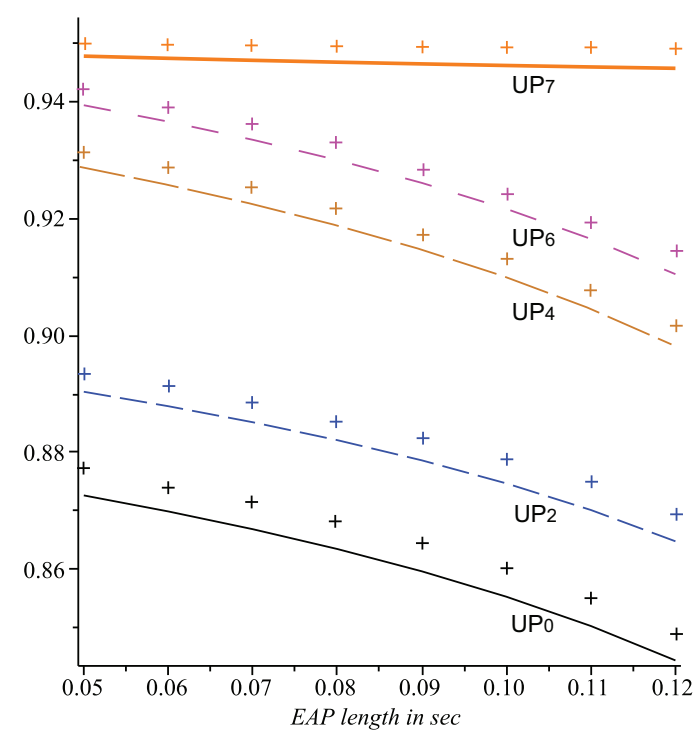

(b) Successful Transmission Probability

Fig. 5. Network performance: all UPs, length of RAP1 is $0.3 \mathrm{sec}$.

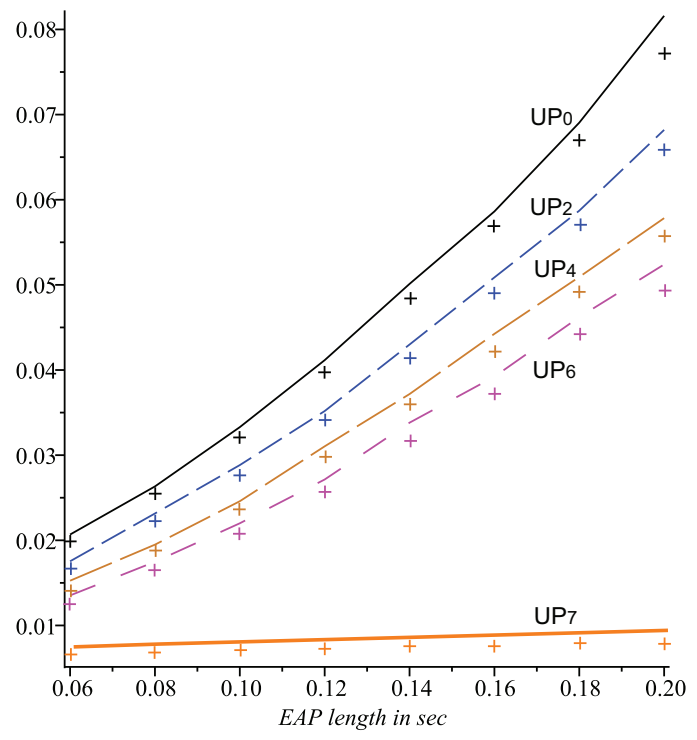

(a) All UPs with four nodes each.

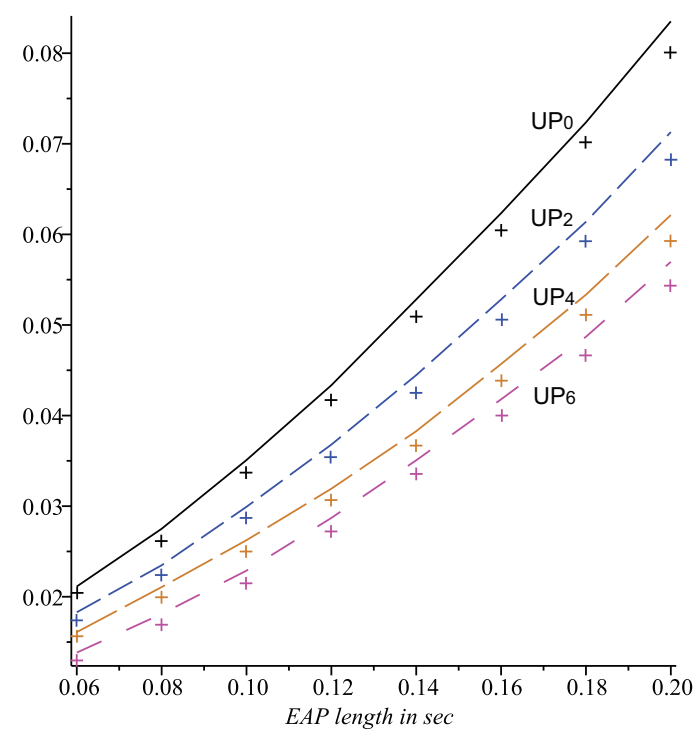

(b) UPs 0,2 , 4, and 6, with 8 nodes each.

Fig. 6. Mean waiting time; length of RAP1 is $0.3 \mathrm{sec}$.

conclude that four priorities are more than enough, and that merging together priorities 0 and 1,2 and 3 , and 4 and 5, respectively, would not change the results in any noticeable way.

Thus, it seems safe to conclude that having four priorities instead of the original eight would give very similar results in terms of performance, while simplifying considerably the design of the firmware and software, as well as reducing the complexity of the hardware.

We have also analyzed network performance in the case where the network has only two UPs with 16 nodes each: one group of nodes with $\mathrm{UP}_{0}$, and another with either $\mathrm{UP}_{6}$ or $\mathrm{UP}_{7}$; the results are shown in Fig. 7. As can be seen, assigning traffic to the highest priority traffic class in case of non-zero EAP access phase, leads to better performance results for all nodes.

\section{SummaRY AND CONCLUSION}

The results shown above allow the following observations to be made.

Short EAP and RAP phases lead to inefficient use of WBAN bandwidth; in particular, slots at the end of EAP phases are wasted since there is not enough time for completing a frame transmission. In addition, frame collision probability noticeably increases at the beginning of a RAP phase because the nodes at all priorities below $\mathrm{UP}_{7}$ have just unlocked 


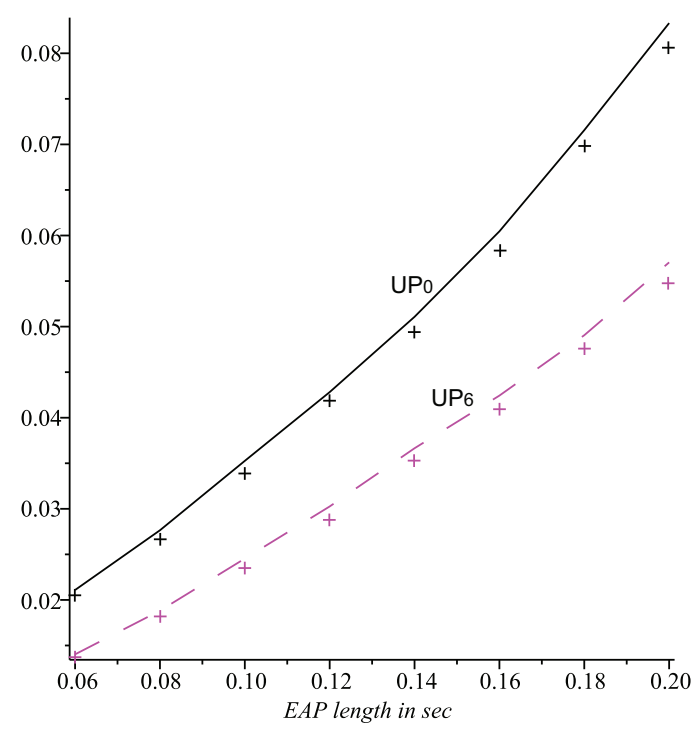

(a) UPs 0 and 6, 16 nodes each.

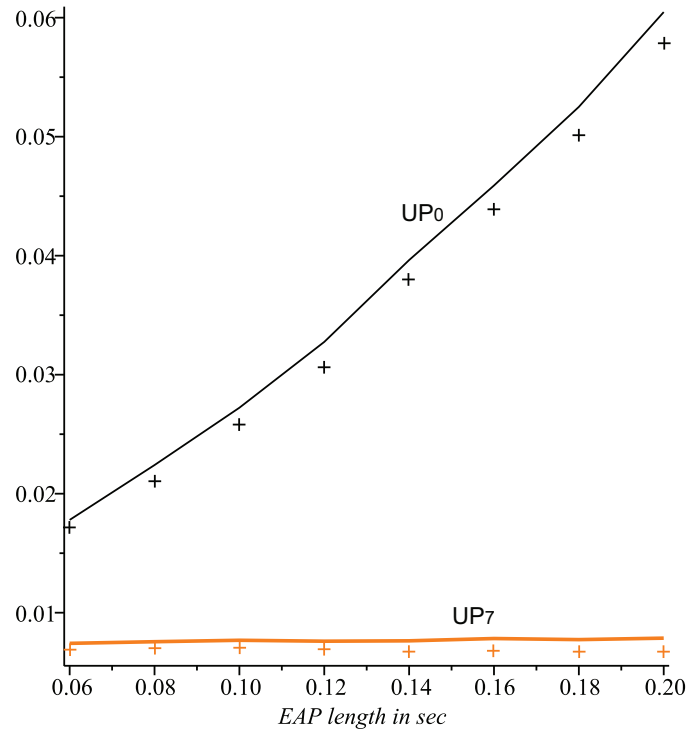

(b) UPs 0 and 7, 16 nodes each.

Fig. 7. Mean waiting time; length of RAP1 is $0.3 \mathrm{sec}$.

their backoff counter and are, thus, highly likely to transmit a data frame. Since these nodes had to pause their backoff counter form the previous RAP and phase, and given the small contention window sizes of WBAN UPs, the number of such nodes is high, and degradation of performance is very likely.

In fact, EAP phases are largely unnecessary as they provide little performance improvement for $\mathrm{UP}_{7}$ nodes, which are already highly prioritized by the small contention window sizes, while considerably decreasing the performance for all other UPs. As the result, overall WBAN performance is degraded unless the network traffic is high - which is not a typical WBAN scenario.

Based on the current contention window sizes for different priority classes, deployment of eight UPs is unnecessary. Our results indicate that traffic classes 0 and 1,2 and 3, and 4 and 5, respectively, have almost equal performance measures. Therefore, satisfactory performance could be obtained with only four priority classes, namely $\mathrm{UP}_{0}, \mathrm{UP}_{2}, \mathrm{UP}_{4}$, and $\mathrm{UP}_{7}$ for WBANs, which could reduce the development and manufacturing costs of IEEE 802.15.6-compliant hardware and software.

Finally, the IEEE 802.15.6 CSMA/CA mechanism is not very efficient in utilizing the medium. In particular, small contention window sizes for all UPs lead to early saturation by increasing the collision probability under medium to high network traffic volume. Increasing the $\mathrm{CW}$ size would decrease the collision probability and, thus, improve improve the WBAN performance at the expense of higher energy consumption.

Finally, the results of the analysis performed in the network that uses RTS/CTS handshake (presented in the Online Supplement) indicate that deploying the RTS/CTS mechanism degrades the network performance, esp. when the WBAN operates well below the saturation regime and data frames of small to medium size are used.

It remains to be seen whether these problem areas will preclude wider utilization of the IEEE 802.15.6 standard for the development of WBANs.

\section{REFERENCES}

[1] M. R. Yuce, "Implementation of wireless body area networks for healthcare systems," Sensors and Actuators A: Physical, vol. 162, pp. 116-129, Jul. 2010.

[2] H. Chen, W. Wu, and J. Lee, "A WBAN-based real-time electroencephalogram monitoring system: Design and implementation," Journal of Medical Systems, vol. 34, pp. 303-311, 2010.

[3] Y.-S. Seo, D.-Y. Kim, J. Cho, and B. Lee, "OCDP: A WBAN MAC protocol for contention-based medical and CE applications," in Proc. the 4th International Conference on Ubiquitous Information Management and Communication, Suwon, Korea, Jan. 2010.

[4] K. S. Kwak, M. A. Ameen, D. Kwak, C. Lee, and H. Lee, "A study on proposed IEEE 802.15 WBAN MAC protocols," in Proc. the 9th international conference on Communications and information technologies, Incheon, Korea, Sep. 2009, pp. 834-840.

[5] F. E. H. Tay, D. G. Guoa, L. Xua, M. N. Nyana, and K. L. Yap, "MEMSWear-biomonitoring system for remote vital signs monitoring," Journal of the Franklin Institute, vol. 346, pp. 531-542, Aug. 2009.

[6] D. Albu, J. Lukkien, and R. Verhoeven, "On-node processing of ECG signals," in Proceedings of the 7th IEEE conference on Consumer communications and networking conference, Las Vegas, Nevada, USA, Jan. 2010, pp. 1165-1169.

[7] Wireless Body Area Networks Standard, IEEE Std. 802.15.6, Feb. 2012.

[8] S. Ullah and K. S. Kwak, "Throughput and delay limits of IEEE 802.15.6," in Proc. IEEE WCNC, Cancun, Mexico, Mar. 2011.

[9] S. Ullah, M. Chen, and K. S. Kwak, "Throughput and delay analysis of IEEE 802.15.6-based CSMA/CA protocol," Journal of Medical Systems, vol. 36, p. 3875-3891, Jul. 2012.

[10] S. Rashwand, J. Mišić, and H. Khazaei, "IEEE 802.15.6 under saturation: Some problems to be expected," Journal of Communications and Networks, vol. 13, pp. 142-149, 2011.

[11] S. Rashwand, J. Mišić, and H. Khazaei, "Performance analysis of IEEE 802.15.6 under saturation condition and error-prone channel," in Proc. the IEEE Wireless Communications and Networking Conference (WCNC'11), Cancun, Mexico, Mar. 2011, pp. 475-480.

[12] S. Rashwand and J. Mišić, "Effects of access phases lengths on performance of IEEE 802.15.6 CSMA/CA," Computer Networks, Vol. 56, pp. 2832-2846, 2012.

[13] F. Daneshgaran, M. Laddomada, F. Mesiti, and M. Mondin, "Unsaturated throughput analysis of IEEE 802.11 in presence of non ideal transmission channel and capture effects," IEEE Transactions on Wireless Communications, vol. 7, pp. 1276-1286, Apr. 2008. 
[14] C.-S. Hwang and J. M. Cioffi, "Opportunistic CSMA/CA for achieving multi-user diversity in wireless LAN," IEEE Transactions on Wireless Communications, vol. 8, pp. 2972-2982, Jun. 2009.

[15] X. Ling, K. H. Liu, Y. Cheng, X. Shen, and J. W. Mark, "A novel performance model for distributed prioritized MAC protocols," in Proc. the IEEE Global Telecommunications Conference (Globecom'07), Washington, DC, US., Nov. 2007, pp. 4692-4696.

[16] O. M. F. Abu-Sharkh and A. H. Tewfik, "Toward accurate modeling of the IEEE 802.11e EDCA under finite load and error-prone channel," IEEE Transactions on Wireless Communications, vol. 7, pp. 2560-2570, 2008.

[17] I. Inan, F. Keceli, and E. Ayanoglu, "Analysis of the 802.11e enhanced distributed channel access function," IEEE Transactions on Communications, vol. 57, pp. 1753-1764, Jun. 2009.

[18] J. Mišić and V. Mišić. Wireless Personal Area Networks Performance, Interconnections and Security with IEEE 802.15.4. Wiley Publishing, Inc., first edition, 2008.

[19] F. Martelli, C. Buratti, and R. Verdone, "On the performance of an IEEE 802.15.6 wireless body area network," in Proc. 11th European Wireless Conference, Vienna, Austria, Apr. 2011, pp. 1-6.

[20] S. Rashwand, J. Mišić, and V. B. Mišić, "MAC performance modeling of IEEE 802.15.6-based WBANs over rician-faded channels," in Proc. the IEEE International Conference on Communications (ICC'12), Ottawa, Canada, Jun. 2012, pp. 5462-5467.

[21] B. H. Jung, R. U. Akbar, and D. K. Sung, "Throughput, energy consumption, and energy efficiency of IEEE 802.15 .6 body area network (BAN) MAC protocol," in IEEE 23rd International Symposium on Personal, Indoor and Mobile Radio Communications - (PIMRC), Sydney, Australia, Sep. 2012, pp. 584-589.

[22] S. Rashwand and J. Mišić, "Performance evaluation of IEEE 802.15.6 under non-saturation condition," in Proc. the IEEE Global Telecommunications Conference (Globecom'11), Houston, Texas, US., Dec. 2011, pp. 1-6.

[23] Opnet Modeler, Opnet Technologies, Inc. Last accessed on September 2012. Bethesda, MD. [Online]. Available: http://www.opnet.com

[24] D. P. Heyman and M. J. Sobel, Stochastic Models in Operations Research, Vol. I: Stochastic Processes and Operating Characteristics. New York: Dover Publications, INC., 2005.

[25] H. Takagi, Queueing Analysis; a Foundation of Performance Evaluation - Volume 1: Vacation and Priority Systems. NORTH HOLLAND, 1991.

[26] H. Takagi, Queueing analysis; a foundation of performance evaluation - volume 3: discrete-time systems. NORTH HOLLAND, 1991.

[27] Maple 13, Maplesoft, Inc. Last accessed on September 2012. Waterloo, Canada. [Online]. Available: http://www.maplesoft.com

[28] J. D. Bronzino, The Biomedical Engineering Handbook - Third Edition. Volume 2: Biomedical Engineering Fundamentals. Taylor \& Francis Group, LLC, 2006.

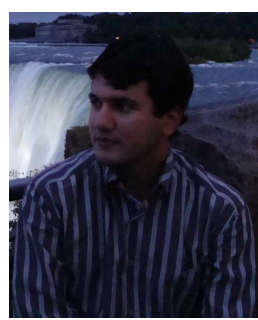

Saeed Rashwand received his $\mathrm{PhD}$ degree in 2012 from University of Manitoba, Winnipeg, MB, Canada, and his MSc degree in 2008 from Amirkabir University of Technology, Iran, both in Computer Science. His current research interests include performance evaluation, design, and implementation of wireless body area networks, wireless sensor networks, mobile ad-hoc networks, and wireless local area networks as well as cloud computing.

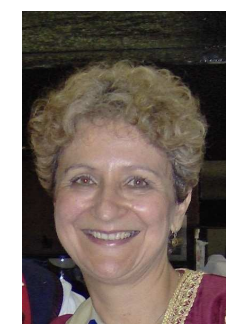

Jelena Mišić (M91, SM08) Professor of Computer Science at Ryerson University in Toronto, Ontario, Canada. She has published over 90 papers in archival journals and more than 120 papers at international conferences in the areas of wireless networks, in particular wireless personal area network and wireless sensor network protocols, performance evaluation, and security. She serves on editorial boards of IEEE Transactions on Vehicular Technology, Computer Networks, Ad hoc Networks, Security and Communication Networks, Ad Hoc \& Sensor Wireless Networks, Int. Journal of Sensor Networks, and Int. Journal of Telemedicine and Applications. She is a Senior Member of IEEE and Member of ACM.

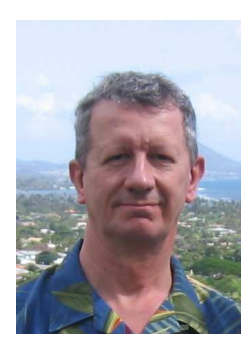

ACM and AIS.
Vojislav B. Mišić is Professor of Computer Science at Ryerson University in Toronto, Ontario, Canada. He received his $\mathrm{PhD}$ in Computer Science from University of Belgrade, Serbia, in 1993. His research interests include performance evaluation of wireless networks and systems and software engineering. He has authored or co-authored six books, 20 book chapters, and over 200 papers in archival journals and at prestigious international conferences. $\mathrm{He}$ is a Senior Member of IEEE, and member of 


\section{Analysis of CSMA/CA mechanism of IEEE 802.15.6 under non-saturation regime: the Impact of RTS/CTS Handshake}

Saeed Rashwand, Jelena Mišić, Senior Member, IEEE, and Vojislav B. Mišić, Senior Member, IEEE

The IEEE 802.15.6 standard does not mention the use of RTS/CTS handshake, but neither does it prohibit it. Therefore, it may be of interest to see whether its use would give any benefits. This Online supplement presents the results of performance evaluation of our model in case RTS/CTS handshake is used. We first present the differences in the analytical model, and then show the results of performance evaluation.

\section{ANALYTICAL MODEL: CHANGES NEEDED TO ACCOMMODATE RTS/CTS HANDSHAKE}

In this section, we will briefly outline the changes that need to be introduced in the analytical model to accommodate the RTS/CTS handshake. Let $r t s$ and $c t s$ represent the size of RTS and CTS (in slots) and $r t s_{b}$ and $c t s_{b}$ (in bits), respectively, and let $\delta=(1-b e r)^{r t s_{b}+c t s_{b}}$ denote the probability that neither RTS nor CTS is corrupted by noise. All other variables are the same as in the main paper.

In Equation (2), the mean number of CSMA slots in the EAP1 period is updated via

$$
X_{E}^{\prime}=\frac{e a p}{\chi+n_{7} \tau_{7} \psi \delta L_{7, s}+\left(1-\chi-n_{7} \tau_{7} \psi \delta\right) L_{7, c}}
$$

where the transmission time for a $\mathrm{UP}_{k}$ data frame in slots is $L_{k, s}=r t s+c t s+l_{k}+a c k+3$ sifs and $L_{k, c}=r t s+c t s+s i f s$ for successful and unsucccesful transmission, respectively. The corresponding transmission times of data frames sent by other nodes are $L_{k, s o}=r t s+c t s+l_{k, o}+a c k+3 s i f s$ and $L_{k, c o}=$ $r t s+c t s+$ sifs, respectively.

In the Markov chain in Fig. 2 in the main file, $\eta_{k}=f_{k} \delta$ represents the probability of successful access to the medium for a $\mathrm{UP}_{k}$ node when its backoff counter reaches zero.

The probability that the queue of the node with $\mathrm{UP}_{k}$ is empty when a data frame is either successfully transmitted or dropped due to an exceeded retry limit is $\pi_{k, 0}=\pi_{k, 0}^{\prime} \sigma_{k}$; it is calculated in the queuing sub-model.

The PGFs for the duration of a successful and unsuccessful data frame transmission, failure being due to a RTS

- S. Rashwand, J. Mišić and V. B. Mišić are with the Department of Computer Science, Ryerson University, Toronto, Ontario.

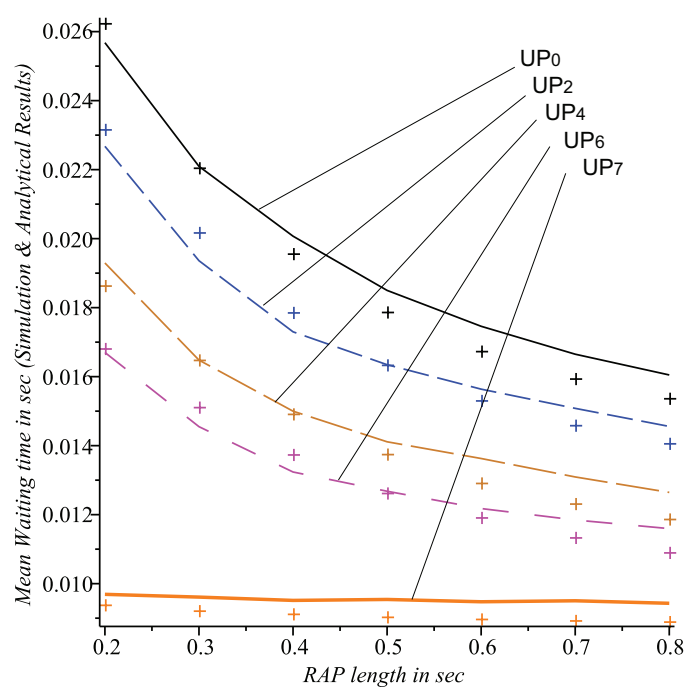

Fig. 1. Mean waiting time in the network with all UPs; length of EAP1 is $0.05 \mathrm{sec}$.

collision, by $\operatorname{St}_{k}(z)=z^{r t s+c t s+l_{k}+a c k+3 s i f s}$ and $C t_{k}(z)=$ $z^{r t s+c t s+s i f s}$, respectively.

The PGF for the service time of a data frame is given by $B_{k}(z)=\frac{\sigma_{k} \Phi_{k}(z) S t_{k}(z)}{1-\left(1-\sigma_{k}\right) \Phi_{k}(z) S t_{k}(z)}$.

The PGF of waiting time for a $\mathrm{UP}_{k}$ data frame, as per equation (25), is modified by multiplying $\Theta_{k}(z)$ from equation (26) by $(r t s+2 s i f s+c t s) z+1-r t s+2 s i f s+c t s$, which corresponds to the PGF for the duration of the RTS/CTS handshake.

\section{Performance of medium access With RTS/CTS}

Once those changes are incorporated in the analytical model, it is solved in the same manner as the one described in the main paper. The simulation and analytical results for the mean waiting time of data frames for all user priorities where the RAP1 length varies while the length of EAP1 is constant are shown in Fig. 1. As before, we have not shown the results for all user priorities in order to improve clarity; the priorities 


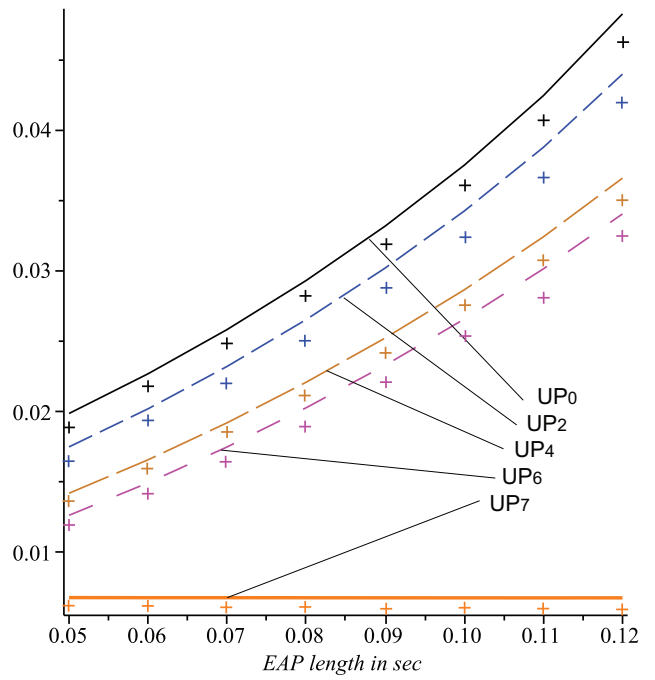

(a) Mean waiting time.

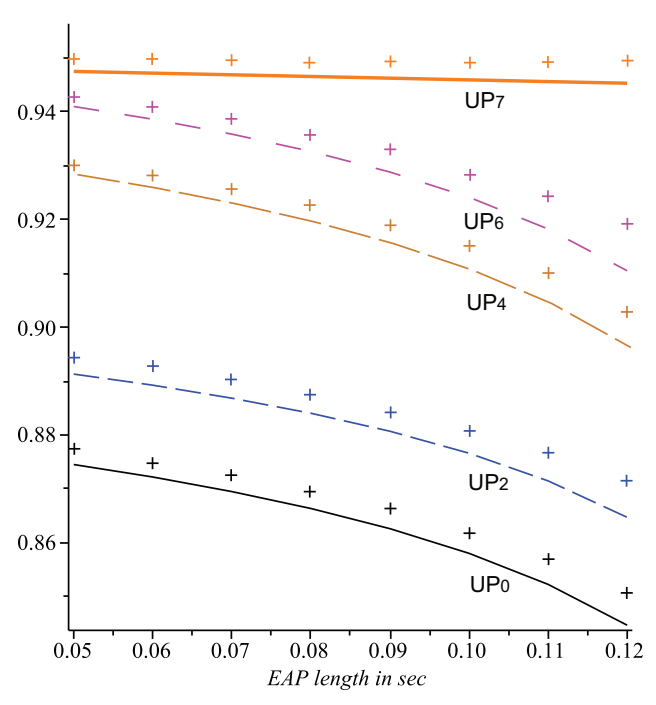

(b) Probability of successful transmission.

Fig. 2. Performance of the network with all UPs; length of RAP1 is $0.3 \mathrm{sec}$.

shown are labeled in the diagrams. Lines denote analytical results, while crosses denote simulation results.

As can be seen, increasing the length of RAP1 while EAP1 is kept constant improves (i.e., reduces) the frame delay for all UPs, since longer RAP periods lead to decrease of collision probability after an EAP period. In addition, having short EAP and RAP periods increases the number of CSMA slots in which the medium cannot be accessed by the nodes because there is not enough time to complete a data frame transaction during the current access phase.

In the second scenario, the length of EAP1 varies from 0.05 second to 0.12 second while the length of RAP1 is set to 0.3 second; the results are shown in Fig. 2, again with lines and crosses denoting analytical and simulation results, respectively.

Comparing the graphs in Figs. 1 and 2 above with their counterparts, Figs. 4 and 5 in the main paper, we see that the behavior of the network does not depend on the use of RTS/CTS - in qualitative terms, that is. With or without RTS/CTS, mean waiting time and transmission success probability of data frames increase for all UPs when the length of EAP1 increases. Namely, having a long EAP period essentially wastes network resources because the traffic load of the highest priority traffic category, $\mathrm{UP}_{7}$, is low. However, during the RAP periods the traffic congestion increases because other nodes have shorter time periods for transmission. The conclusion is that judicious choice of RAP and EAP periods according to the traffic loads of the UPs should lead to substantial performance improvements.

However, the difference in quantitative terms is quite noticeable, as the performance of the WBAN improves dramatically when the RTS/CTS mechanism is not used, esp. in terms of waiting time which is about 50\% lower when RTS/CTS is not used. The probability of successful transmission is only slightly higher when RTS/CTS is used, probably because the original values were high enough, so there's not much improvement there to justify the use of RTS/CTS handshake.
We may conclude that the use of RTS/CTS is counterproductive in the scenario shown, where small to moderate data frame sizes of up to the maximum payload size of $600 \mathrm{~B}$ are used. This setup was selected for use in an environments where signal to noise ratio (SNR) is low, in which case larger payloads are more likely to result in frequent frame errors and retransmissions.

However, for a WBAN in an environment with sufficiently high SNR, larger frame sizes may be used. In this case, using the RTS/CTS mechanism improves the performance of the network because the RTS/CTS avoids many of the errors, and its transmission time is considerably smaller than the transmission time of the data frame itself.

\section{SUMmary}

Our results indicate that, for an unsaturated WBAN with a small to moderate data frame sizes, deploying the RTS/CTS mechanism degrades the network performance compared to the case where the nodes immediately transmit their data frames upon a successful medium access. Since the control frames are not much smaller in size compared to the data frames, there seems to be no benefit in using RTS/CTS; on the contrary, it actually degrades network performance. 